\title{
Regulation of Spine Calcium Dynamics by Rapid Spine Motility
}

\author{
Ania Majewska, Ayumu Tashiro, and Rafael Yuste \\ Department of Biological Sciences, Columbia University, New York, New York 10027
}

\begin{abstract}
Dendritic spines receive most excitatory inputs in the CNS and compartmentalize calcium. Spines also undergo rapid morphological changes, although the function of this motility is still unclear. We have investigated the effect of spine movement on spine calcium dynamics with two-photon photobleaching of enhanced green fluorescent protein and calcium imaging of action potential-elicited transients in spines from layer $2 / 3$ pyramidal neurons in mouse visual cortex slices. The elongation or retrac-
\end{abstract}

tion of the spine neck during spine motility alters the diffusional coupling between spine and dendrite and significantly changes calcium decay kinetics in spines. Our results demonstrate that the spine's ability to compartmentalize calcium is constantly changing.

Key words: GFP; imaging; two photon; photobleaching; LTP; neocortex
As first predicted by Ramón y Cajal (1891), dendritic spines receive most synaptic inputs in the mammalian CNS (Gray, 1959; Harris and Kater, 1994). Spines are separated from their parent dendrites by a thin neck and compartmentalize calcium during synaptic stimulation (Müller and Connor, 1991; Yuste and Denk, 1995; Yuste et al., 2000). Calcium compartmentalization in spines is likely to be functionally important, because calcium mediates input-specific forms of synaptic plasticity (Lynch et al., 1983; Malenka et al., 1989). Calcium decay kinetics in spines is controlled by diffusion of calcium across the spine neck and active removal of calcium from the spine cytoplasm (Majewska et al., 2000a) as well as by calcium buffers endogenous to the spine head. Therefore, the morphology of the spine neck and the expression and regulation of calcium pumps and buffers control the duration of calcium transients in spines.

Spines have been shown recently to be extremely motile on the timescale of seconds (Fischer et al., 1998; Dunaevsky et al., 1999). The function of spine motility is still unclear. Although new filopodia and spines can appear after electrical stimulation (Engert and Bonhoeffer, 1999; Maletic-Savatic et al., 1999), basal motility, such as that present in the absence of stimulation, appears resilient to major changes in the activity of the cell (Dunaevsky et al., 1999). Both spine and filopodial motility declines with development (Dailey and Smith, 1996; Ziv and Smith, 1996; Dunaevsky et al., 1999) and is thought to be related to critical periods of synaptic rearrangements (Dunaevsky et al., 1999; Lendvai et al., 2000). Finally, volatile anesthetics block spine motility, suggesting that rapid motility may play a global role in brain function (Kaech et al., 1999).

The finding that calcium decays in spines are regulated by calcium diffusion through the spine neck (Majewska et al., 2000a) suggests that spine motility could alter this diffusional coupling and potentially modify spine calcium decay kinetics. On the other hand, calcium pumps at the spine (Majewska et al., 2000a) and endogenous calcium buffers also control decay kinetics, and the type and amount of motility could be too small to produce significant changes in these dynamics.

We have explored the effect of spine motility on calcium dynamics by the use of two-photon excitation to photobleach enhanced green fluorescent protein (EGFP) and image calcium indicators in motile spines from layer $2 / 3$ pyramidal neurons in slices of mouse

Received July 18, 2000; revised Aug. 28, 2000; accepted Aug. 29, 2000.

This work was funded by the National Eye Institute Grant EY 111787 and the Human Frontier Science Program. We thank E. Brown, A. Dunaevsky, J. Goldberg, K. Holthoff, and C. Mason for comments.

Correspondence should be addressed to Dr. Ania Majewska, Department of Biological Sciences, Columbia University, 1212 Amsterdam Avenue, Box 2435, New York, NY 10027. E-mail: akm21@columbia.edu.

Copyright (C) 2000 Society for Neuroscience $0270-6474 / 00 / 208262-07 \$ 15.00 / 0$ visual cortex. We find that the diffusion of EGFP across the spine neck is linearly correlated with the length of the spine neck and is altered during motility. We also find that changes in spine neck length affect the initial fast calcium decay in spines. Our findings show that spine motility alters calcium decay kinetics and demonstrate, for the first time, that spine motility has an effect on spine function. We hypothesize that, by altering spine-dendrite coupling, rapid spine motility serves to alter constantly spine calcium dynamics and therefore continuously changes the spine's capacity as a calcium compartment and, presumably, changes the implementation of local learning rules.

\section{MATERIALS AND METHODS}

Slices and electrophysiology. All experiments were performed in accordance with the National Institutes of Health Guide for the Care and Use of Laboratory Animals (NIH publication no. 86-23; revised 1987). Cultured slices were prepared from the primary visual cortex of postnatal day 0 (P0)-P1 C57 mice as described (Dunaevsky et al., 1999), incubated for 2 weeks, and biolistically transfected with cytomegalovirus-EGFP vector (Clontech, Palo Alto, CA). After 2-4 d, slices were imaged in artificial CSF (ACSF) that contained (in $\mathrm{mM}$ ): $126 \mathrm{NaCl}, 3 \mathrm{KCl}, 2 \mathrm{CaCl}_{2}, 2 \mathrm{MgSO}_{4}$, $1.1 \mathrm{NaH}_{3} \mathrm{PO}_{4}, 26 \mathrm{NaHCO}_{3}$, and 10 dextrose, bubbled with $95 \% \mathrm{O}_{2}$ and $5 \%$ $\mathrm{CO}_{2}$. Acute slices were made from primary visual cortex from P12 to P16 mice as described (Peterlin et al., 2000). Layer $2 / 3$ pyramidal neurons in V1 were selected under differential interference contrast, and whole-cell recordings were made with an Axoclamp 2B (Axon Instruments, Foster City, CA) amplifier. Borosilicate pipettes with an outer diameter of $1.5 \mathrm{~mm}$ and an inner diameter of $0.86 \mathrm{~mm}$ were used. The intracellular solution was (in $\mathrm{mM}): 5 \mathrm{NaCl}, 10 \mathrm{KCl}, 10 \mathrm{HEPES}, 135 \mathrm{KMeSO}_{4}, 2.5-4 \mathrm{Mg}$-ATP, 0.3 Na-GTP, and 1-5 mM calcium green-1 (Molecular Probes, Eugene, OR); resistances were 6-7 M $\mathrm{M}$. Electrophysiological signals were digitized by the use of an analog-to-digital board and Superscope (InstruNet; GW Instruments, Somerville, MA). Action potentials were elicited by stimulating the cell extracellularly with a patch pipette containing ACSF and positioned close to the base of the soma. Stimulating currents were in the range of $0.5-1 \mathrm{~mA}$ and were elicited with an IsoFlex stimulator (AMPI).

Two-photon imaging and photobleaching. Imaging and photobleaching were done by the use of a custom-made two-photon laser-scanning microscope (Majewska et al., 2000b) consisting of a modified confocal microscope (Fluoview; Olympus, New Hyde Park, NY) and a titanium-sapphire laser providing $130 \mathrm{fsec}$ pulses at $75 \mathrm{MHz}$ at wavelengths of $740-850 \mathrm{~nm}$ (Mira; Coherent, Palo Alto, CA) pumped by a solid-state source (Verdi; Coherent). A $60 \times, 0.9$ numerical aperture water immersion objective (LUMPlanFl/IR1; Olympus) was used. Fluorescence was detected by the use of a photomultiplier tube (HC125-02; Hamamatsu) in external, wholearea detection mode, and images were acquired and analyzed with Fluoview (Olympus) software. A Pockels cell (model 350-50; ConOptics, Danbury, CT) was used to create brief $(\sim 3-20 \mathrm{msec})$ pulses of high-intensity illumination for measurements of diff usional times across the spine neck and to block the beam during times in the scan when data were not being collected. Spines were chosen randomly from all areas of the cell in a 200 $\mu \mathrm{m}$ radius from the soma-including spines on basal, apical, and oblique dendrites. In the current configuration our microscope has the resolution (1/e radius) of $0.3 \mu \mathrm{m}$ in the radial dimension and $1 \mu \mathrm{m}$ in the $z$ dimension (point spread function measured with subresolution beads). Images of spines were acquired at the highest digital zoom $(10 \times)$, resulting in a 
nominal spatial resolution of 30 pixels/ $\mu \mathrm{m}$ and in a time resolution of 12.5 $\mathrm{msec} /$ point in line-scan mode. For each imaged spine, a $z$-stack at intervals of $0.1 \mu \mathrm{m}$ was taken to allow a careful reconstruction of spine morphology, and photobleaching or calcium measurements were performed. After 5-30 min, the procedure was repeated on the same spine. To determine spine motility indexes, time-lapse sequences were taken every 30-60 sec. At each time point, five to nine focal planes $0.5-1 \mu \mathrm{m}$ apart were scanned; these were later projected into a single image. Because spine motility is temperature dependent, experiments were performed at room temperature to allow all data to be collected before a spine changed shape. Control measurements were taken at $37^{\circ} \mathrm{C}$ and yielded correlations that agreed with the room temperature data ( $n=3$ spines for calcium; $n=10$ for GFP measurements); both sets of measurements were therefore pooled for the correlation of the changes in neck length and changes in diffusion time constants, whereas only room temperature data were used in the correlation of neck length and time constants.

Analysis. Fluorescence signals were analyzed with Igor (Wavemetrics, Lake Oswego, OR) as described previously (Majewska et al., 2000a). Two to four traces were averaged for analysis of $\tau_{\mathrm{GFP}}$, whereas three to seven traces were averaged for measurements of calcium decay kinetics. Fluorescence intensity was corrected for background fluorescence measured in an area adjacent to the structure and presented either as the change in fluorescence divided by the initial fluorescence recorded before the stimulus was applied $(\Delta F / F)$ or as total fluorescence divided by the initial fluorescence $\left(F / F_{\text {initial }}\right) . \Delta F / F$ and $F / F_{\text {jnitial }}$ decays were fitted to single or multiple exponentials. Spine calcium decays were mostly double exponential, and the time constant of the first fast exponential was determined by fixing the second slow time constant to that of the parent dendrite. No difference was observed in traces analyzed without assuming any time constant for the second decay phase (Majewska et al., 2000a). In some cases in which the dendrite did not undergo a significant calcium increase, the spine appeared to be single exponential, and the time constant of this exponential was used. Morphological measurements, image processing, and analysis were done with custom-written macros using NIH Image as described (Dunaevsky et al., 1999). Images were aligned to correct for drift in the $x-y$ plane. For calculations of the motility index, images were thresholded to a single level throughout the entire sequence. Images were then binarized, and the spines were outlined. The motility index (Dunaevsky et al., 1999) is a measure of the space a spine occupies as it moves over time. It is defined as the ratio of the difference between accumulated and smallest areas occupied by the spine divided by the average area of the spine, when the outlines of the spine in a time-lapse recording were superimposed digitally:

$$
\text { Motility index }=\frac{(\text { accumulated area }- \text { smallest area })}{\text { average area }} .
$$

Spine neck lengths were measured from $z$-stack images. In most cases the spine necks were clearly visible and could be followed from the spine to the dendrite. In some cases the spine necks were too dim to be seen. In these cases the position of the spine neck was inferred from the shape of the spine and was taken to start at the point in the spine head where the morphology deviated from a spherical shape. It is possible in these cases that the true length of the spine neck was underestimated if the spine neck was curved. To minimize the error caused by angled spine necks, only spines in the same $z$ plane as the middle of the dendrite or spines with spine necks that were clearly visible in a single $z$ section were analyzed.

\section{RESULTS}

\section{Changes in spine neck length during spine motility}

We first characterized the effects of spine motility on the length of the spine neck by using a custom-built two-photon microscope to image layer 2/3 pyramidal neurons from mouse V1 cultured slices transfected with EGFP (Fig. $1 A, B$ ). Spines on apical, oblique, and basal dendrites were imaged in time-lapse sequences and found to be highly motile over periods of minutes. As described (Fischer et al., 1998; Dunaevsky et al., 1999), morphological changes were diverse and included morphing, growth and retraction, development of filopodia, and appearance and disappearance of spines. We analyzed the changes in spine neck lengths, finding large changes during imaging sequences lasting $15 \mathrm{~min}$ (Fig. 1C). Spine neck changes ranged from 20 to $250 \%$ of the initial length. The average maximal changes in spine neck length were $80 \pm 15 \%$ of the starting length in spines (mean \pm SEM for all data; $n=15$ from 2 neurons). We concluded that spine neck length is constantly changing in spines.

\section{Diffusional coupling between spine and dendrite depends on the spine neck length}

Diffusion across the spine neck can be modeled as diffusion through a pipe. The rate of diff usional equilibration between spine
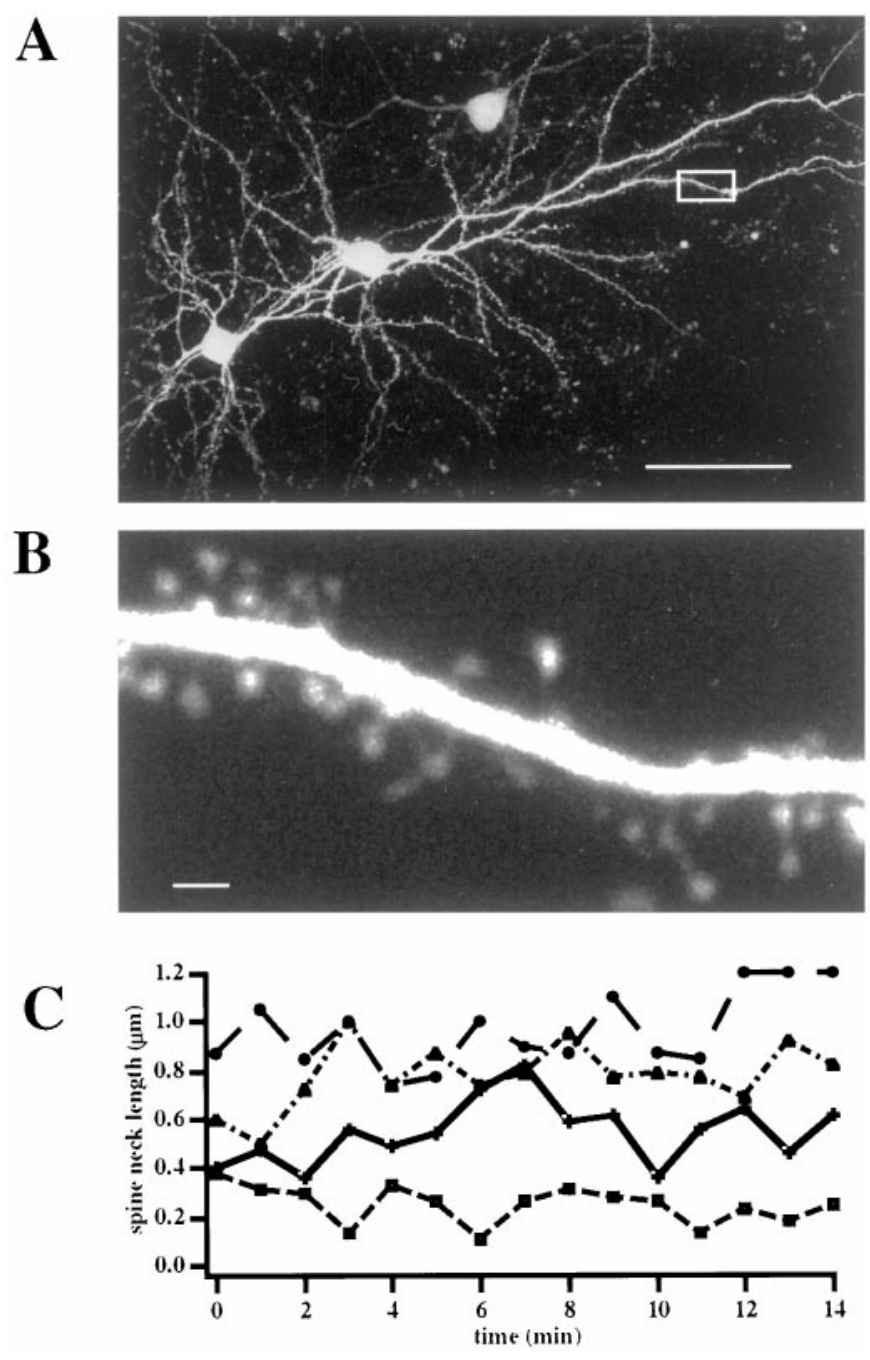

Figure 1. Spine neck lengths change during spine movement. $A$, Twophoton image of two EGFP-transfected layer $2 / 3$ pyramidal neurons. The pial surface is to the top right. $B$, High-magnification image of the boxed area in $A$. Note how dendritic spines are clearly visible. C. Spine necks elongate and retract as they move. Analysis of the spine neck changes in four spines followed for a $15 \mathrm{~min}$ period. Scale bars: $A, 50 \mu \mathrm{m} ; B, 1 \mu \mathrm{m}$.

and dendrite should depend on morphological features such as spine neck length, spine neck diameter, and spine head volume. The equation that governs the relationship between morphological parameters and the timescale of diffusion through a pipe and, under our assumptions, between spine and dendrite is:

$$
\tau_{\text {dif usion }}=\frac{l \cdot V}{D \cdot \pi \cdot r^{2}},
$$

where $l$ is the length of the spine neck, $V$ is the volume of the spine head, $D$ is the diffusion coefficient of the diff using molecule, and $r$ is the radius of the spine neck. Because long-necked spines tend to have slower diffusion of fluorescein dextran across the spine neck than do short-necked spines (Svoboda et al., 1996), we focused on the length of the spine neck and measured the diffusion rates of EGFP across the neck in spines with necks of different lengths. For these experiments, EGFP in a spine head was bleached by the use of a brief (3-20 msec; approximate spine exposure of $500 \mu \mathrm{sec}$ to 1 $\mathrm{msec}$ ) pulse of high-intensity illumination in line-scan mode, and the fluorescence recovery caused by the diffusion of unbleached fluorophore (Axelrod et al., 1976) from the dendrite was measured with lower intensity excitation (Svoboda et al., 1996; Majewska et al., 2000a) (Fig. 2). We found that the EGFP fluorescence recovery trace was well fitted with a single exponential with an average $\tau$ of 


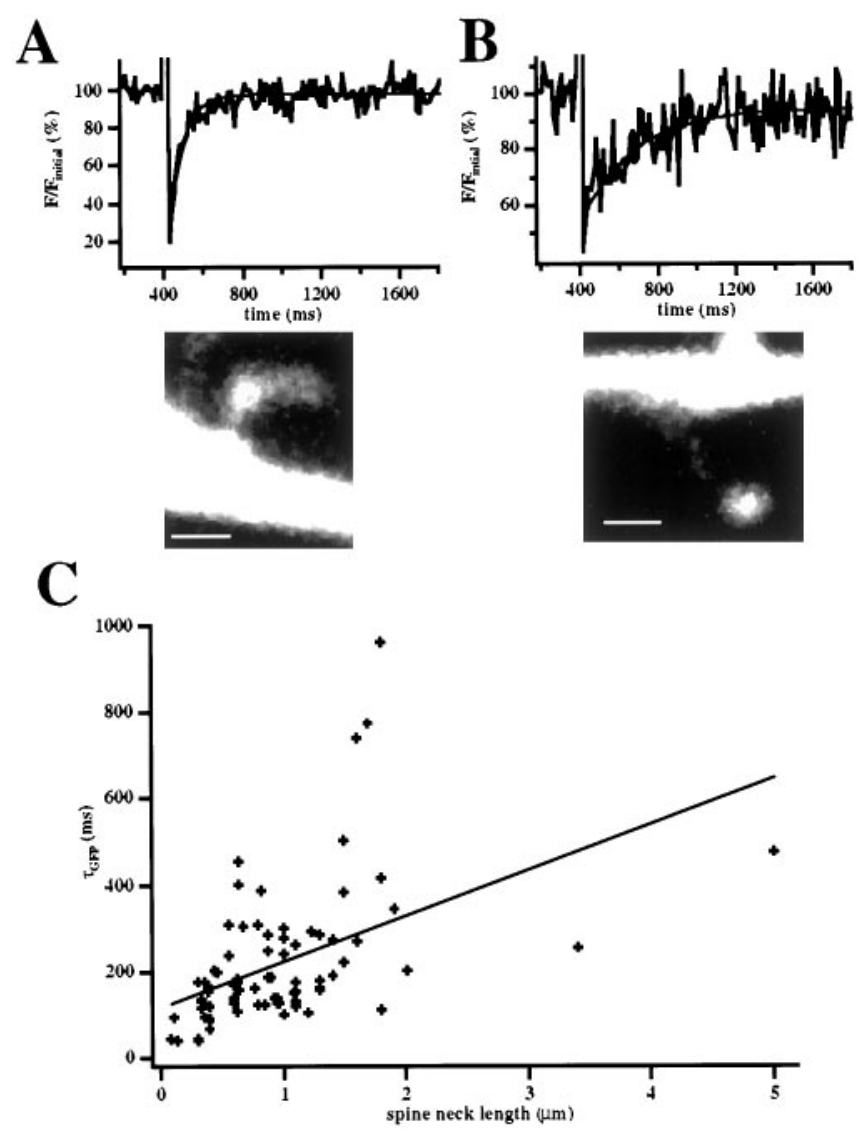

Figure 2. Diff usional coupling between spine and dendrite depends on the length of the spine neck. $A, B$, Measurements of diffusional coupling in short $(A)$ - and long $(B)$-necked spines. $A$, Top, Fluorescence recovery curve of the spine shown in the bottom panel, whose neck length is $0.4 \mu \mathrm{m}$. The fitted monoexponential curve (thin line) shows a $\tau_{\mathrm{GFP}}$ of $68 \mathrm{msec}$. B, Top, Similar measurement from a long-necked $(1.5 \mu \mathrm{m})$ spine, with a $\tau_{\mathrm{GFP}}$ of 275 msec. $C$, Relation of $\tau_{\mathrm{GFP}}$ and spine neck length. There is a good correlation between these two variables. Scale bars, $1 \mu \mathrm{m}$.

$220 \pm 18 \mathrm{msec}(n=72)$. In addition, short spines tended to have faster recoveries than did long-necked spines (Fig. $2 A, B$ ). In fact, across all spines there was a significant correlation between spine neck length and the EGFP fluorescent recovery time constant $\left(\tau_{\mathrm{GFP}} ; p<0.0001\right.$; correlation coefficient $=0.53 ; n=72$; Fig. $\left.2 C\right)$. This implies that spine neck length determines the diff usional coupling between spine and dendrite. Although it is possible that spine neck length correlates with another parameter such as neck diameter or the viscosity or obstruction of the spine neck, such correlations were not found in statistical studies using EM (Harris and Stevens, 1989). Therefore, we think it likely that the relationship between spine neck length and EGFP diffusion is causal.

\section{Diffusional coupling between spine and dendrite changes as spines move}

After establishing that spine neck length is correlated with diffusional coupling and that spine neck changed during spine motility, we examined whether diffusional coupling is also altered during motility. We first determined whether the bleaching procedure altered spine motility because exposure to high-intensity light could cause injury to the cell. For this purpose we measured the motility of spines on a dendritic branch before and after a small percentage of these spines was bleached. We used the motility index (Dunaevsky et al., 1999) (MI; see Materials and Methods) to measure spine motility. Bleached spines did not show a significant difference in the motility index before $(1.34 \pm 0.33)$ and after $(1.34 \pm 0.11)$ the bleaching procedure $(n=5$; Fig. $3 A)$. In addition, spines that had not undergone the bleaching procedure but were on the same dendritic branch also did not significantly change their motility
A

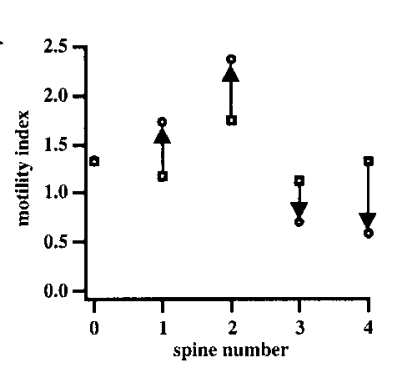

B

\section{C}

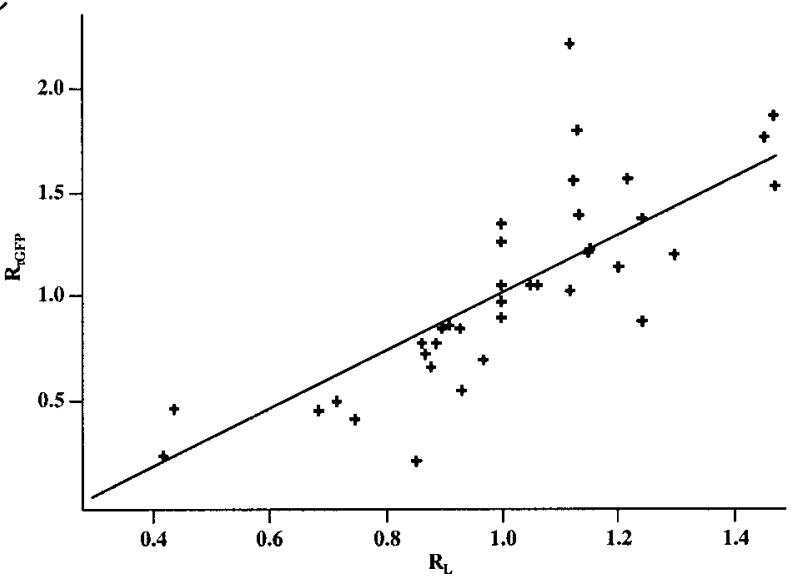

Figure 3. Diffusional coupling between spine and dendrite is altered as spines elongate or retract. $A, B$, Lack of effect of the bleaching protocol on the spine motility of bleached $(A)$ or unbleached $(B)$ spines on the same dendrite. Each panel shows the motility index of spines before (squares) and after (circles) the bleaching protocol was applied. There were no significant differences in the average motility indexes. $C$, Relation between the $R_{\tau \mathrm{GFP}}$ (change in fluorescent recovery time constant) and the $R_{L}$ (change in spine neck length). Notice the strong correlation between the two parameters $(p<0.0001 ; n=38$; linear regression; correlation coefficient $=0.73)$ indicating that changes in the times for diffusion of EGFP through the spine neck are tied to the changes in spine neck length during motility.

after photobleaching $(1.37 \pm 0.26$ before bleaching; $1.46 \pm 0.16$ after bleaching; $n=6 ; p=0.74$; Fig. $3 B)$. We concluded that the bleaching did not alter spine motility on the bleached dendrite.

We then compared the fluorescence recovery of EGFP in individual spines at different time points, after the spines showed significant changes in neck length. Observed changes in spine neck length ranged from 0 to $250 \%$. To measure the change in spine neck length, we used the ratio $R_{L}$, where $R_{L}$ is defined as the neck length at the current time point divided by the neck length measured at the time point directly before it (typically $15 \mathrm{~min}$ ). Similarly, $R_{\tau \mathrm{GFP}}$ was defined as $\tau_{\mathrm{GFP}}$ at the current time point divided by the $\tau_{\text {GFP }}$ measured at the preceding time point. Thus, a value of 1 for either of these ratios signifies no change in either length or $\tau_{\mathrm{GFP}}$. In fact, we found a strong correlation between the change in neck length $\left(R_{L}\right)$ of individual spines and the change in $\tau_{\mathrm{GFP}}(p<$ 0.0001 ; correlation coefficient $=0.73 ; n=38$ from 26 spines; Figs. $3 C$, 4). Most spines that did not change neck length also did not change $\tau_{\mathrm{GFP}}$ (Fig. $4 A$ ). This shows that the spine's diffusional coupling to the dendrite and therefore its ability to act as a separate biochemical compartment change during motility.

\section{The spine calcium decay kinetics is proportional to the spine neck length}

We then wondered whether changes in spine neck length during spine movement translated to changes in the regulation of calcium decay kinetics in spines. To answer this, we imaged calcium dynamics in spines from neurons in acute slices filled with calcium green, using the calcium transient elicited by backpropagating action potentials (APs) (Yuste and Denk, 1995) as a stereotyped 


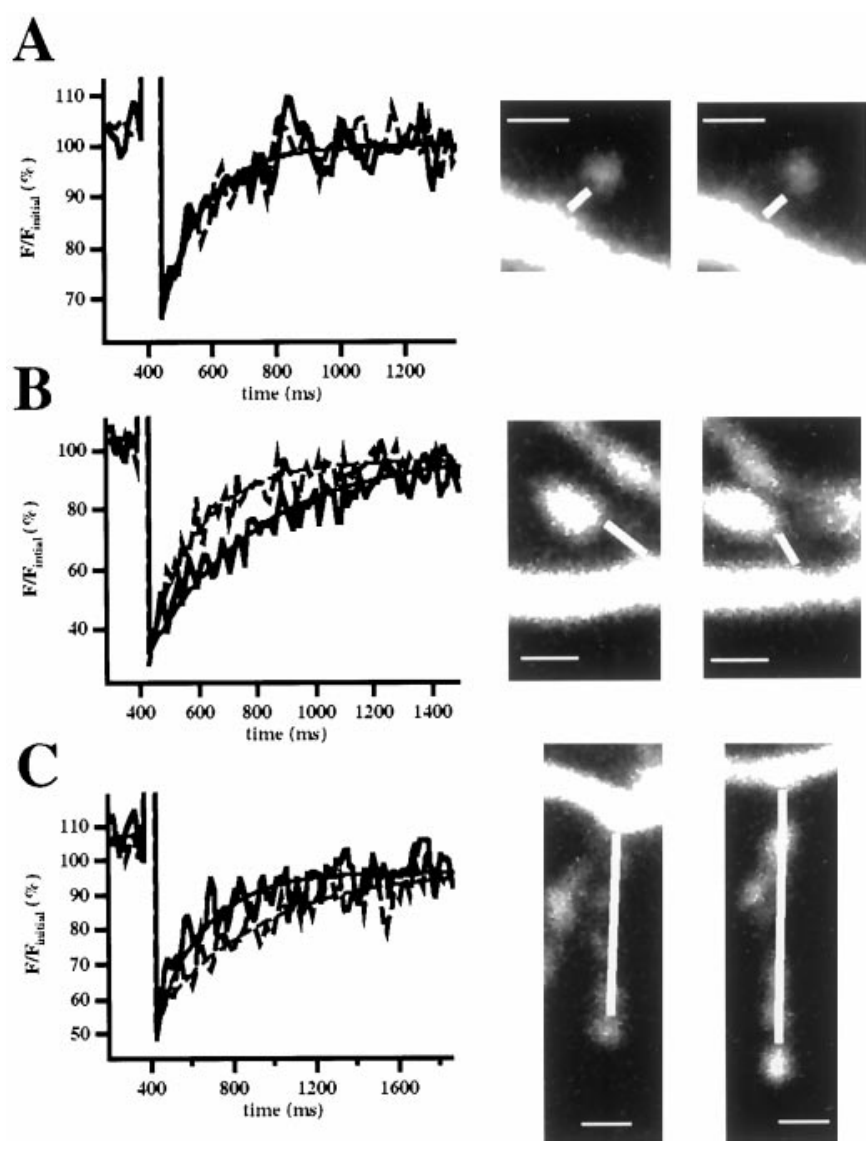

Figure 4. Changes in EGFP diffusion during movement in individual spines. $A-C$, Measurements of diffusional coupling in spines whose necks stayed the same length $(A)$, shortened $(B)$, or grew longer $(C)$. Middle, Right, Images of the spine at the first time point (middle) and at the second time point (right). White lines trace the length of the neck. Left, The EGFP fluorescence recovery traces. Solid traces correspond to the recoveries obtained at the first time point (middle), whereas stippled traces correspond to those obtained at the second time point (right). The change in time constants for the spines shown is as follows: from 130 to $135 \mathrm{msec}(A)$, from 380 to $170 \mathrm{msec}(B)$, and from 260 to $480 \mathrm{msec}(C)$. Scale bars, $1 \mu \mathrm{m}$.

delta function to monitor the timescales of calcium decays in spines during motility.

Spine motility is actin-based (Fischer et al., 1998; Dunaevsky et al., 1999) and is blocked by prolonged perfusion of the cell cytoplasm during whole-cell recording (Dunaevsky et al., 1999). To avoid "washing out" spine motility, we patched pyramidal neurons with high concentrations of dye (a bolus injection) and withdrew the pipette within 30-90 sec of the break-in. This bolus injection (Helmchen et al., 1996) allowed us to fill the cell with concentrations of dye sufficient for the discrimination of spines and the imaging of calcium transients while circumventing the need for sustained whole-cell recording. Indeed, spines on control neurons that were whole-cell recorded for prolonged periods of time with $200 \mu \mathrm{M}$ calcium green were almost completely stationary $(\mathrm{MI}=$ $0.7 \pm 0.1 ; n=7$ spines from 2 cells), whereas spines on bolus-filled cells were highly motile $(\mathrm{MI}=1.9 \pm 0.2 ; n=10$ spines from 2 cells).

After filling the neurons with the bolus injection and waiting 30 min to allow the dye to equilibrate throughout the neuron, we stimulated the neuron with a pipette placed close to the base of the soma. AP firing was inferred from generalized calcium increases in spines and dendrites (Yuste and Denk, 1995). In control experiments the pipette was not withdrawn, and we confirmed that indeed the stimulation caused the cell to fire single APs $(n=3)$.

We found that in response to single APs, spines from bolusinjected cells had double-exponential decay kinetics (Majewska et al., 2000a) (Fig. $5 A)$ with a fast first decay $\left(\tau_{\text {fast }}=298 \pm 20 \mathrm{msec}\right.$;
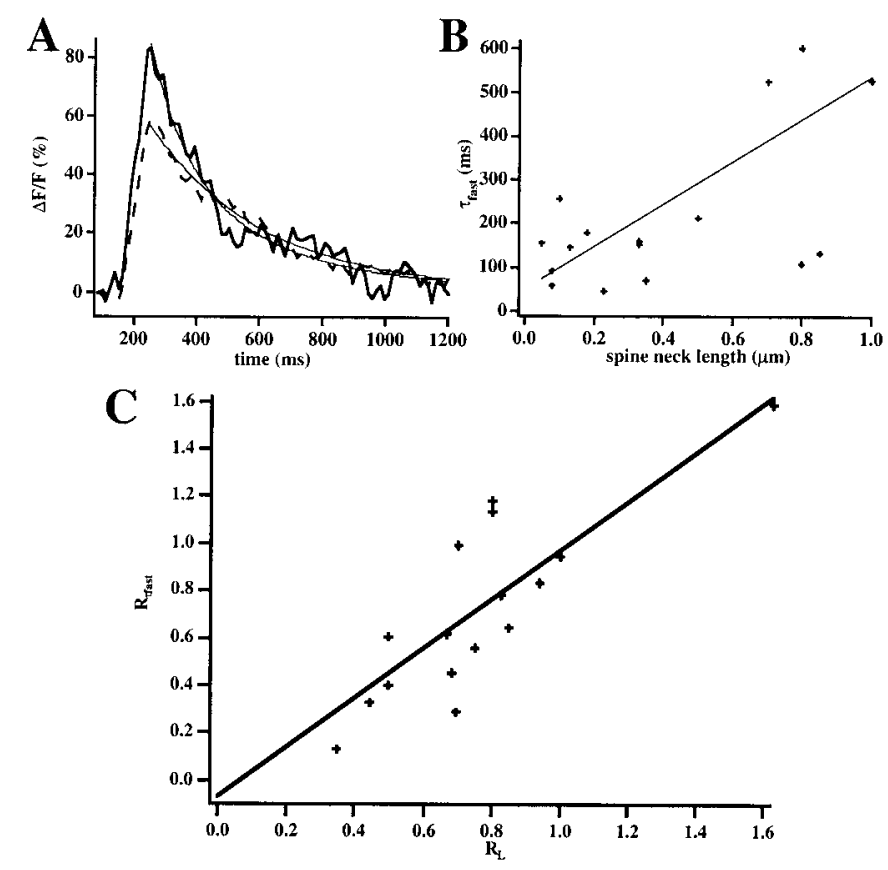

Figure 5: Correlation between changes in the spine neck length and changes in the fast calcium decay in spines. $A$, Calcium dynamics in a spine after the firing of a backpropagating action potential through an extracellular pipette placed near the cell soma. The action potential elicits an increase in calcium concentration in both dendrite and spine. The calcium decay in the dendrite (stippled line) follows single-exponential kinetics, whereas the decay in the spine (solid line) shows the characteristic doubleexponential kinetics (Majewska et al., 2000a). The trace is an average of six trials, filtered with a seven-point smoothing kernel. $B$, Relation between spine $\tau_{\text {fast }}$ (fast time constant of decay) and spine neck length. There is a strong correlation between these two variables $(p<0.01$; correlation coefficient $=0.66 ; n=16)$. $C$, Relation between $R_{\text {ffast }}$ and $R_{L}$. Note the strong correlation between these parameters $(p<0.001 ; n=16$; correlation coefficient $=0.83$ ) demonstrating that changes in spine neck length during spine movement affect the kinetics of the calcium decay in spines.

$n=22$ ), intrinsic to the spine (Majewska et al., 2000a), and a second decay with a slower timescale determined by the parent dendrite $\left(\tau_{d}=590 \pm 116 \mathrm{msec} ; n=7\right)$. As expected (Majewska et al., 2000a), the first fast decay scaled with the length of the spine neck $(p<0.01$; correlation coefficient $=0.66 ; n=16$; Fig. $5 B)$. Thus, spines with longer necks had slower calcium decay kinetics.

\section{Calcium decays in spines change during spine movement}

The observed correlations predicted that the changes in spine neck length occurring during spine movement would alter the first fast decay. Indeed, we observed that the initial fast decay kinetics of spines was not stable over time as compared with that of their parent dendrites. Although dendritic decay time constants fluctuated by $<10 \%$ over time, spine kinetics changed by as much as $60 \%$ (SDs for spine-dendrite pairs over a period of $30 \mathrm{~min}$ were $7 \pm 1 \%$ for dendrites vs $38 \pm 5 \%$ for spines; $n=9$ from 4 cells). Nevertheless, other factors besides the spine neck length can control spine decay kinetics. In particular, spine decay kinetics is also regulated by spine calcium pumps (Majewska et al., 2000a), as well as other morphological factors such as the neck diameter or blocking of the neck by the spine apparatus (Gray, 1959), which could potentially have a large effect on spine calcium decay kinetics.

To explore whether changes in spine neck length produce corresponding changes in spine calcium decays, we performed sequential calcium imaging from spines for up to $30 \mathrm{~min}$ and correlated their fast decay kinetics with simultaneous measurements of their spine neck length (10 spines from 6 neurons). Most of the imaged spines retracted, and only one spine elongated. It is possible that we preselected for longer spine necks as these are easier to distinguish with calcium green-filled neurons that are dimmer than GFP- 


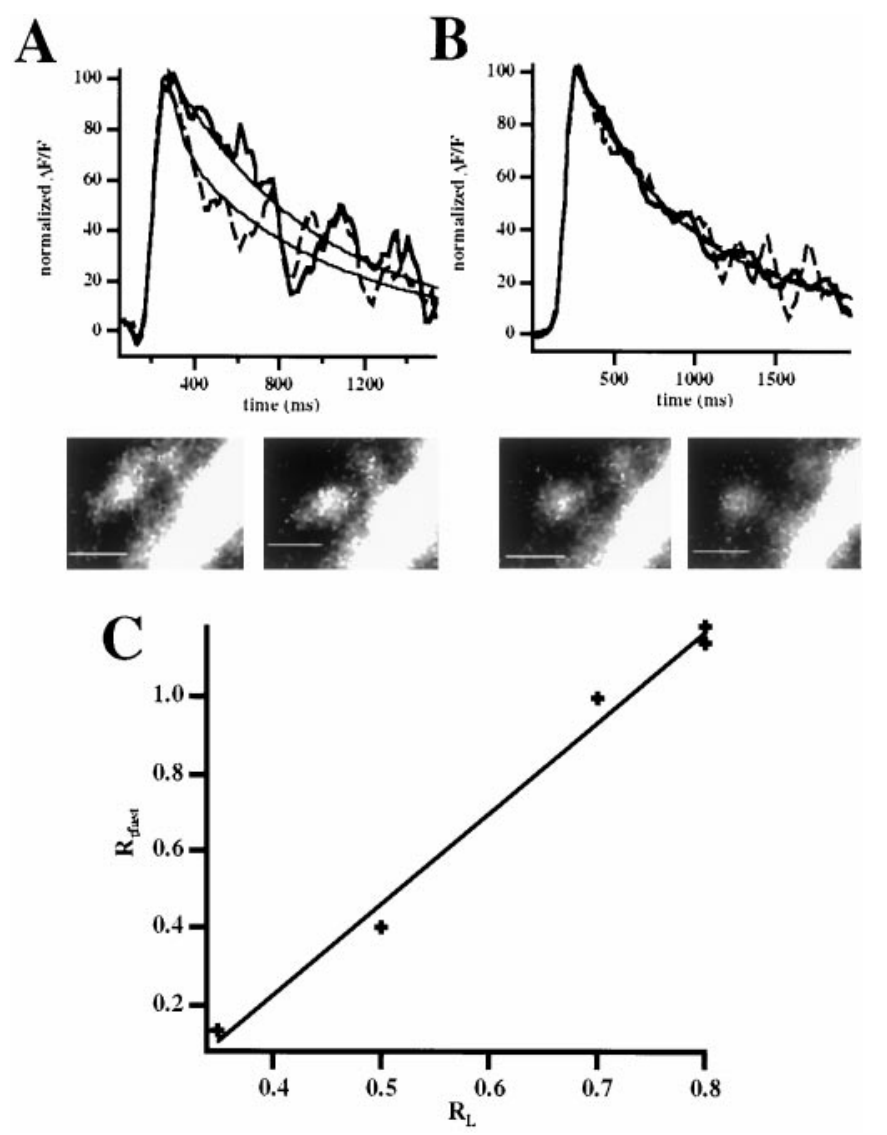

Figure 6. Changes in the fast calcium decay component during spine movement in individual spines. $A$, Example of the calcium dynamics in a spine in response to an AP. Two calcium decays from the same spine measured at different time points (top) are shown. The traces have been normalized to the same amplitude for ease of comparison. The solid line shows the decay that corresponds to the bottom left image of the spine. The stippled line corresponds to the bottom right image when the spine had retracted and the spine neck had become shorter. Notice the faster initial decay of the stippled trace. The second slow decay phase is similar in the two spines because of the slower and less variable dendritic decay. $B$, Similar analysis from a spine that did not change its neck length. Note how the calcium decay kinetics is very similar. $C$, Relation between $R_{\tau \text { fast }}$ and $R_{L}$ for a spine in which five time points were available. The correlation is highly significant $(p<0.005 ; n=5)$. Scale bars, $1 \mu \mathrm{m}$.

transfected cells. We found a strong correlation between the change in calcium decay kinetics $\left(R_{\text {fast }}\right)$ and the change in the length of the spine neck $\left(R_{L} ; p<0.005\right.$; correlation coefficient $=$ $0.83 ; n=16$; Fig. $5 C$ ). The linear fit through the data yielded a slope close to 1 , as expected (slope $=1.04$, linear regression; see Eq. 1).

Changes in calcium decay kinetics with motility were also evident in individual spines, with decays becoming faster as spines retracted and becoming slower as spine necks lengthened (Fig. 6A). In a spine in which five time points were available, the correspondence between changes in decay kinetics and changes in neck length was also significant $(p<0.005 ; n=5$; Fig. $6 B)$. We concluded that changes in the spine neck length because of rapid spine motility affect calcium decay kinetics.

\section{DISCUSSION}

\section{Spine motility regulates spine calcium compartmentalization}

Morphological changes in spine shape in animals subjected to many different experimental paradigms have been well documented (Valverde, 1967; Purpura, 1974; Fifkova and Anderson, 1981; Brandon and Coss, 1982), including changes in the length of the spine neck (Fifkova and Anderson, 1981; Brandon and Coss, 1982).
In 1977, actin was purified in the postsynaptic density and was proposed to underlie spine motility (Blomberg et al., 1977). A similar proposal was later made by Crick, who predicted the existence of an actin-based "twitching" of spines (Crick, 1982). Indeed, rapid motility of filopodia (Dailey and Smith, 1996; Ziv and Smith, 1996) and spines (Fischer et al., 1998; Dunaevsky et al., 1999) has been found recently throughout the CNS. Spine motility changes during critical periods for monocular deprivation in mouse area V1 (Dunaevsky et al., 1999) and can be regulated by sensory deafferentation (Lendvai et al., 2000), and new protrusions can grow in response to synaptic stimulation (Engert and Bonhoeffer, 1999; Maletic-Savatic et al., 1999). This suggests that spine motility is related to critical period plasticity. Nevertheless, spine motility persists in the absence of synaptic stimulation (Dunaevsky et al., 1999) and appears intrinsic to the neuron (Dunaevsky et al., 1999). Thus, spine motility remains an orphan phenomenon without a clearly demonstrated function.

Although some studies suggest that motility may allow spines to find synaptic partners (Dailey and Smith, 1996; Ziv and Smith, 1996), we show here, for the first time, that spine motility has an effect on the spine's function as a calcium compartment: changes in spine neck length that occur during motility produce changes in calcium decay kinetics. This is important because the main specific function of dendritic spines could be to compartmentalize calcium and implement local learning rules (Müller and Connor, 1991; Yuste and Denk, 1995; Yuste et al., 2000). We concentrated on the study of spine neck length although other morphological parameters such as spine head volume and spine neck radius that might be altered during motility could also have an effect on spine function. By measuring the diffusion of EGFP across the spine neck in transfected neurons, we find that as spines retract and shorten their spine necks, diffusion between the spine and dendrite becomes faster. Conversely, when spines elongate, diff usion between the two structures becomes slower. By measuring AP-induced calcium transients in neurons injected with calcium green-1, we determine whether calcium kinetics is also affected during motility. We show that as spines retract they maintain high calcium concentrations for shorter periods of time as indicated by their faster initial decays and become less able to serve as calcium compartments. Therefore, as spines move, their ability to act biochemically independently of the dendrite is altered.

Interestingly, small changes in neck length can cause large changes in the decay kinetics of spines, especially if the initial length of the spine neck is small. Stubby spines, which were undersampled in this study because of their small size and proximity to the dendrite, could experience major changes in calcium dynamics after minuscule changes in neck length. In fact recent studies have shown that spine categories such as stubbies, thin and mushroom spines, and filopodia are not stable and that considerable conversions between these categories take place (Parnass et al., 2000). This suggests that changes in decay kinetics are more profound than those documented here, especially in the case in which stubby spines change into thin or mushroom spines.

\section{Comparisons with previous studies}

Previously Svoboda et al. (1996) studied the diff usion of fluorescein dextrans between spine and dendrite by bleaching the dye or uncaging it in spines. In a statistical population, the authors found that diffusion time increased in longer necked spines although direct correlations between neck length and diffusion times were not presented. Our study documents changing diffusion times as the neck lengths change during single-spine elongation and retraction. Although the diffusion coefficients of fluorescein dextran and EGFP in cytoplasm are similar (Popov and Poo, 1992; Swaminathan et al., 1997), we find diffusion times a factor of two to three larger than that reported in the previous study, even in populations of spines with similar neck lengths. This could be caused by differences in the preparation used or by differences in spine geometries-such as spine neck diameter or spine head volume. Also, the extensive whole-cell recordings necessary to introduce 
fluorescein dextran into the neurons could have reduced the neurons' viscosity.

As opposed to fluorescein dextran and EGFP diffusion, the dynamics of calcium in neurons is subject to complicated regulation that is still not well understood (Neher, 1998). Our study builds on our previous work that has described heterogeneity in the calcium decay dynamics in spines (Majewska et al., 2000a). Here we show that the static heterogeneity described in our previous study is a dynamic process in which spines do not maintain their calcium kinetics but change between the various extremes that were described. There are important methodological differences between our studies. Our first study was performed in rat CA1, whereas this study is done in mouse V1. Also our first study used prolonged whole-cell recordings, whereas in this work we use brief whole-cell recordings. This method does not wash out the cellular contents as profoundly as prolonged whole-cell recording, and therefore we expect that most of the spine's mobile calcium buffers remain intact. In this present study, we again find that the spine decay kinetics is double exponential. This argues that our previous work describing the mechanisms of the decay kinetics is not seriously affected by the potential washout of endogenous buffers.

\section{Other factors may regulate spine calcium decays}

Spine calcium decays are shaped by many factors such as calcium extrusion mechanisms that contribute to both phases of the decay (Majewska et al., 2000a). Extrusion rates may be regulated in time either by inactivation mechanisms (Wang et al., 1991) or by increased expression, and these processes may be calcium dependent (Guerini et al., 1999). Endogenous calcium buffers also shape calcium kinetics by slowing down extrusion-based timescales (Tank et al., 1995) and altering diffusional ones depending on their mobility (Gabso et al., 1997). Endogenous buffers in pyramidal neurons have been shown to be relatively immobile and appear to have low buffer capacities and affinities (Helmchen et al., 1996), suggesting that they slow diff usion and do not saturate unless trains of stimuli are presented. We find no changes in extrusion or buffering mechanisms at the timescales we have explored although it is possible that longer timescales or different stimulation paradigms could reveal this regulation.

\section{Mechanisms of changes in spine neck length}

The major effect of changes in spine neck length on spine biochemical compartmentalization draws attention to the molecular mechanisms that might control this process and their regulation. Indeed, we have found evidence recently of the involvement of the Rho family of small GTPases in the control of the spine neck length (Tashiro et al., 2000). The Rho family is implicated in the regulation of the actin cytoskeleton in a variety of cell types, including neurons (Hall, 1994; Luo et al., 1996; Threadgill et al., 1997). In our study in hippocampal pyramidal neurons overexpression of a constitutively active form of Rho significantly reduced the length of the spine neck. At the same time, expression of C3 transferase, which is analogous to a dominant-negative Rho, significantly increased the spine neck length. Therefore, the ability of the spine to compartmentalize calcium is directly regulated by this GTPase. It thus becomes interesting to explore the regulation of the expression and activity of Rho in neurons, as well as the effects of this regulation on the function of the circuit and behavior of the animal.

\section{Implications for synaptic function and plasticity}

The modulation of calcium decay kinetics that we observe could have a large impact on the function of the synapse. Spines appear to be ideally built to compartmentalize calcium (Wickens, 1988; Holmes, 1990; Koch and Zador, 1993; Yuste et al., 2000), and the regulation of $\left[\mathrm{Ca}^{2+}\right]_{\mathrm{i}}$ dynamics in spines could underlie synapsespecific learning rules (Levy and Steward, 1979; Gustafsson and Wigstrom, 1986; Lisman, 1989). The changes in kinetics that we measure can be several fold in $30 \mathrm{~min}$. Therefore major changes in the integrated $\left[\mathrm{Ca}^{2+}\right]_{\mathrm{i}}$ experienced by a spine occur as a consequence of its motility. This implies that the same paradigm that elicits a type of synaptic plasticity may not be effective in the same synapse a few minutes later. We would predict that synapses on spines are constantly changing the time window for their calciumdependent learning rules. This effect would not occur on synapses made directly on the dendritic shaft, frequent on interneurons (Peters et al., 1976). The purpose of this fluid change in spine time constants is enigmatic, but it may provide a highly adaptive mechanism to ensure flexible temporal integration of signals.

\section{REFERENCES}

Axelrod D, Koppel D, Schlessinger J, Elson E, Webb W (1976) Mobility measurement by analysis of fluorescence photobleaching recovery kinetics. Biophys J 16:1055-1069.

Blomberg F, Cohen R, Siekevitz P (1977) The structure of postsynaptic densities isolated from dog cerebral cortex. II. Characterization and arrangement of some of the major proteins within the structure. J Cell Biol 86:831-845.

Brandon J, Coss R (1982) Rapid dendritic spine stem shortening during one-trial learning: the honeybee's first orientation flight. Brain Res 252:51-61.

Crick F (1982) Do spines twitch? Trends Neurosci 5:44-46.

Dailey ME, Smith SJ (1996) The dynamics of dendritic structure in developing hippocampal slices. J Neurosci 16:2983-2994.

Dunaevsky A, Tashiro A, Majewska A, Mason CA, Yuste R (1999) Developmental regulation of spine motility in mammalian CNS. Proc Natl Acad Sci USA 96:13438-13443.

Engert F, Bonhoeffer T (1999) Dendritic spine changes associated with hippocampal long-term synaptic plasticity. Nature 399:66-70.

Fifkova E, Anderson CL (1981) Stimulation-induced changes in dimensions of stalks of dendritic spines in the dentate molecular layer. Exp Neurol 74:621-627.

Fischer M, Kaech S, Knutti D, Matus A (1998) Rapid actin-based plasticity in dendritic spine. Neuron 20:847-854

Gabso M, Neher E, Spira ME (1997) Low mobility of $\mathrm{Ca}^{2+}$ buffers in axons of cultured Aplysia neurons. Neuron 18:473-481.

Gray EG (1959) Electron microscopy of synaptic contacts on dendritic spines of the cerebral cortex. Nature 183:1592-1594.

Guerini D, Garcia-Martin E, Gerber A, Volbracht C, Leist M, Merino C, Carafoli E (1999) The expression of plasma membrane $\mathrm{Ca}^{2+}$ pump isoforms in cerebellar granule neurons is modulated by $\mathrm{Ca}^{2+}$. J Biol Chem 274:1667-1676.

Gustafsson B, Wigstrom H (1986) Hippocampal long-lasting potentiation produced by pairing single volleys and brief conditioning tetani evoked in separate afferents. J Neurosci 6:1575-1582.

Hall A (1994) Small GTP-binding proteins and the regulation of the actin cytoskeleton. Annu Rev Cell Biol 10:31-54.

Harris KM, Kater SB (1994) Dendritic spines: cellular specializations imparting both stability and flexibility to synaptic function. Annu Rev Neurosci 17:341-371.

Harris KM, Stevens JK (1989) Dendritic spines of CA1 pyramidal cells in the rat hippocampus: serial electron microscopy with reference to their biophysical characteristics. J Neurosci 9:2982-2997.

Helmchen F, Imoto K, Sakmann B (1996) $\mathrm{Ca}^{2+}$ buffering and action potential-evoked $\mathrm{Ca}^{2+}$ signaling in dendrites of pyramidal neurons. Biophys J 70:1069-1081.

Holmes W (1990) Is the function of dendritic spines to concentrate calcium? Brain Res 519:338-342.

Kaech S, Brinkhas H, Matus A (1999) Volatile anesthetics block actinbased motility in dendritic spines. Proc Natl Acad Sci USA 96:10433-10437.

Koch C, Zador A (1993) The function of dendritic spines-devices subserving biochemical rather than electrical compartmentalization. J Neurosci 13:413-422.

Lendvai B, Stern E, Chen B, Svoboda K (2000) Experience-dependent plasticity of dendritic spines in the developing rat barrel cortex in vivo. Nature 404:876-881.

Levy WB, Steward O (1979) Synapses as associative memory elements in the hippocampal formation. Brain Res 175:233-245.

Lisman J (1989) A mechanism for the Hebb and anti-Hebb processes underlying learning and memory. Proc Natl Acad Sci USA 86:9574-9578.

Luo L, Hensch T, Ackerman L, Barbel S, Jan L, Jan YN (1996) Differential effects of the Rac GTPase on Purkinje cell axons and dendritic trunks and spines. Nature 379:837-840.

Lynch G, Larson J, Kelso S, Barrionuevo G, Schottler F (1983) Intracellular injections of EGTA block induction of hippocampal long-term potentiation. Nature 305:719-721.

Majewska A, Brown E, Ross J, Yuste R (2000a) Mechanisms of calcium decay kinetics in hippocampal spines: role of spine calcium pumps and calcium diff usion through the spine neck in biochemical compartmentalization. J Neurosci 20:1722-1734.

Majewska A, Yiu G, Yuste R (2000b) A custom-made two-photon microscope and deconvolution system. Pflügers Arch, in press.

Malenka RC, Kauer JA, Perkel DJ, Nicoll RA (1989) The impact of postsynaptic calcium on synaptic transmission-its role in long term potentiation. Trends Neurosci 12:444-450.

Maletic-Savatic M, Malinow R, Svoboda K (1999) Rapid dendritic mor- 
phogenesis in CA1 hippocampal dendrites induced by synaptic activity. Science 283:1923-1927.

Müller W, Connor JA (1991) Dendritic spines as individual neuronal compartments for synaptic $\mathrm{Ca}^{2+}$ responses. Nature 354:73-76.

Neher E (1998) Usefulness and limitations of linear approximations to the understanding of $\mathrm{Ca}^{2+}$ signals. Cell Calcium 24:345-357.

Parnass Z, Tashiro A, Yuste R (2000) Analysis of spine morphological plasticity in developing hippocampal pyramidal neurons. Hippocampus, in press.

Peterlin ZA, Kosloski J, Mao B, Tsiola A, Yuste R (2000) Optical probing of neuronal circuits with calcium indicators. Proc Natl Acad Sci USA 97:3619-3624.

Peters A, Paley SL, Webster HdF (1976) The fine structure of the nervous system. Philadelphia: Saunders.

Popov SV, Poo MM (1992) Diffusional transport of macromolecules in developing nerve processes. J Neurosci 12:77-85.

Purpura D (1974) Dendritic spine "dysgenesis" and mental retardation. Science 186:1126-1128.

Ramón y Cajal S (1891) Significación fisiológica de las expansiones protoplásmicas y nerviosas de la sustancia gris. Congreso médico valenciano. June 24.

Svoboda K, Tank DW, Denk W (1996) Direct measurement of coupling between dendritic spines and shafts. Science 272:716-719.

Swaminathan R, Hoang CP, Verkman AS (1997) Photobleaching recovery and anisotropy decay of green fluorescent protein GFP-S65T in solution and cells: cytopastic viscosity probed by green fluorescent protein and rotational diffusion. Biophys J 72:1900-1907.

Tank DW, Regehr WG, Delaney KD (1995) A quantitative analysis of presynaptic calcium dynamics that contribute to short-term enhancement. J Neurosci 15:7940-7952.

Tashiro A, Minden A, Yuste R (2000) Regulation of dendritic spine morphology by the Rho family of small GTPases: antagonistic roles of Rac and Rho. Cereb Cortex 10:927-938.

Threadgill R, Bobb K, Ghosh A (1997) Regulation of dendritic growth and remodeling by Rho, Rac, and Cdc42. Neuron 19:625-634.

Valverde F (1967) Apical dendritic spines of the visual cortex and light deprivation in the mouse. Exp Brain Res 3:337-352.

Wang KK, Du YS, Diglio C, Tsang W, Kuo TH (1991) Hormone-induced phosphorylation of the plasma membrane calcium pump in cultured aortic endothelial cells. Arch Biochem Biophys 15:103-108.

Wickens J (1988) Electrically coupled but chemically isolated synapses: dendritic spines and calcium in a rule for synaptic modification. Prog Neurobiol 31:507-528.

Yuste R, Denk W (1995) Dendritic spines as basic units of synaptic integration. Nature 375:682-684.

Yuste R, Majewska A, Holthoff K (2000) From form to function: calcium compartmentalization in dendritic spines. Nat Neurosci 3:653-659.

Ziv N, Smith S (1996) Evidence for a role of dendritic filopodia in synaptogenesis and spine formation. Neuron 17:91-102. 